\title{
Application of Fabric in Uniform Design and Its Development Research
}

\author{
Canming $\operatorname{Liu}^{1, a},{ }^{*}$ Fei Bian ${ }^{2, b}$ \\ ${ }^{1,2}$ College of Fashion Art Design, Donghua University, Shanghai 200051, China \\ abbianfei526@163.com \\ Corresponding author: Bian Fei
}

Key words: uniform design; costume fabric; functionality; trend

Abstract:The uniform design is significantly different from other categories of costume design, and the uniform design is mainly based on the industrial characteristics and principles, i.e., the uniform must adequately reflect and adapt to the characteristics of work environment, object and target. While fabric is one of the most practical elements during uniform design, which is also an element directly connected to the design cost, therefore, we can see the importance of fabric during the execution phase of uniform design. In the meantime, various companies also have increasingly higher requirement for the comfort, safety, health and environment friendliness of fabric, and the knowledge and application of new material has also become a development trend in uniform design.

\section{Basis of fabric selection in uniform design \\ Utility function}

The usage rate of uniform is higher than the general costume, so the highly comfortable fabric can bring better experience to the user, which can also promote the work efficiency in a certain way. For example, in hot summer, first of all, the fabric shall have great breathability to release the hot humidity, the fabric with poor quality will affect the perspiration of sweat, and the skin metabolite will stimulate the skin to make it itchy, which might even cause skin inflammation. In addition, the uniform design for special professions has higher requirement of the fabric, and in addition to the function of comfort, the fabric should also be able to protect the personal safety of staff. For example, for the equipotential screen clothes which the electrician wears under hot-line work, the fabric must be made with the twist yarn combining the relatively expensive tussah silk, tussah yarn and metal wire, which has dense structural organization, and the electrician can wear them to conduct hot-line work under220,000-500,000 volts high-voltage power line.

\section{Price and cost}

Fabric is one major component of the uniform cost, due to different appearances and 
ingredients of material, the prices are also significantly different, and the fabric with same color but different fabrics could have a price difference of several multiples. During purchase of uniform products, the corporate customers tend to prefer "low-cost operation". Therefore, the selection of uniform material is the start to control the cost of uniform product. Of course, this does mean that the poorer the uniform material is, the better, but means that efforts should be made to reduce the cost within the overall design scale, and as a result, the final uniform product will have certain competitiveness in price. Therefore, the price cost is the external restraint during the selection of uniform fabric.

\section{Visual aesthetics}

In different uniform design plans, the material will be selected in accordance with different situations of the project, which have different requirement for the quality and appearance of fabric. In accordance with the uniform design, efforts should not only be made to reasonably choose and use various features of fabric, such as its sensuality, practicality and form maintenance, but various textural effects and aesthetics presented by the fabric surface should also be studied to combine the textural effect aesthetics of uniform, in this way to improve the fabric quality. Therefore, the visual aesthetics is a design requirement during the selection of uniform fabric.

\section{Application of material in uniform design}

\section{Seasonal characteristics}

In the uniform industry, people usually divide the uniform into three seasons: summer, spring \& autumn and winter. The company generally distributes the uniform in accordance with different posts based on the three seasons, the uniform in different season will use different fabric, and the designer should understand and grasp the characteristics and application rules of these fabrics.

\section{Summer fabric}

The summer uniform generally uses the fabric with strong breathability, and the fabric with cotton content is most frequently used in the summer uniform. Although the cotton fabric is soft and fitting with great moisture absorption ability and breathability, it also has the shortages of the tendency to shrink and wrinkle, it isn't very stiff and smooth in appearance, you have to iron it before wearing, it is not very convenient for daily care, and it cannot satisfy the specific requirement of uniform, so it isn't suitable to be used to make uniform. If a proper portion of polyester can be added to cotton, it can not only carry out the advantage of polyester, but also the merits of cotton fabric, the final product will have good flexibility and abrasion resistance, which 
has a stable size and a small shrinkage rate, and it has the characteristics of stiffness, no tendency of wrinkle, easiness of washing and quick drying. As a result, the overall quality of uniform will be improved, and in addition, some chemical fiber and slim worsted fabrics are also commonly used in the design of summer uniform.

\section{Spring \& autumn fabric}

There are various kinds of spring \& autumn fabrics, which have significant difference in price, and it should be selected in accordance with the uniform styles of different companies and the economic situation. The all-wool fabric is not convenient for care, and some fabrics with cotton, wool and chemical fiber contents are all great choices as the fabric of spring \& autumn uniform. For example, the special silk brocade has a smooth and delicate surface, soft touch, great drapability as well as natural and soft color, and the suit uniform made of this fabric looks high grade; the wool-polyester fabric has blended the wool and polyester yarns, which has an expensive price, and it is the top choice of fabric to make high-end suit uniform. In the fabric, the proportion of wool is generally $10 \%-45 \%$, in this way, it can not only maintain the merits of wool fabric, but also carry out the advantages of polyester, it is not easy to deformed, and it also has a smooth texture and elegant appearance, which has a dignified and classic style.

\section{Winter fabric}

The winter uniform fabric is mainly used in the coat, which should have great heat retention property, and the wool fabric and the cotton and down feather filling materials are the most frequently used. Of course, they are also generally more expensive than the summer and spring\& autumn uniform fabrics. With the progress and development of technology, the fabric has become more advanced, and the lighter, slimmer and warmer filling material has become new choice as the fabric of winter uniform.

\section{Job nature}

Based on the job nature, the uniform can be divided into the three categories of professional uniform, professional overall and professional protection suit, and for these three kinds of uniforms, the fabric selections are also different in accordance with different work environments.

\section{Fabric of professional uniform}

The users of professional uniform include: companies with business nature (factory, shopping mall, airport, bank investment, tourism, catering, entertainment, hotel, etc.); law enforcement, administrative and security departments (army, police, court, customs, tax department, etc.); public and non-profits organizations (scientific research, education, student, hospital, etc.). These 
industries have a lot of opportunities to contact the outside world, many positions are the windows of foreign services, and the selection of uniform fabric should be suitable for the status and job of the staff. For example, for hotel uniform, the three-star and five-star hotels have different standards for the uniform fabric, and at the same hotel, the uniforms for the two positions of General Manager and Reception also have different requirements of fabric. Therefore, the selection of professional uniform fabric should comprehensively consider multiple factors, including appearance, cost, function, position characteristics, department characteristics and industrial characteristics.

\section{Fabric of professional overall}

The users of professional overall include the front-line production workers and outdoor workers. They mainly conduct manual labor, there is abrasion between body and costume, they tend to sweat during the work, and the outdoor workers are also under the sunshine and ultraviolet radiation. Due to these practical reasons, many fabrics used in professional uniform are not suitable to make professional overall, the selection of professional overall fabric should not only consider the professional activity of the user, but also satisfy the requirement of special work environment, such as safety and comfort, and the characteristics of fabric usability should be emphasized. For example, for the uniform of electrician and repairman, the dense and thick fabric with great humid absorption, breathability, high resistance to wrinkle, dirt and abrasion and anti-static performance is generally selected.

\section{Professional protection suit}

The users of professional protection suit mainly include the personnel working in some special environments, such as the space, nuclear reactor and biochemical laboratory, which especially emphasizes the protection of personal safety. Therefore, the selection and R \& D (research and development) is not what any one uniform company can entirely grasp, and its development, use and improvement require a long-term process. For example, since the first spacesuit was introduced in the United States in 1961, after gradual improvement, now, the spacesuit consists of 7 layers at torso, and the thickest saddlebag consists of 20 layers, including the cotton comfort layer after special anti-static processing, the rubber backup air impervious liner, the main air impervious liner with composite joint structure, the confinement layer of polyester fabric, the thermal-protective layer which realizes thermal protection through heat reflection and the outside protective layer.

\section{Custom-made fabric}

Although there are thousands of categories and colors of fabric in the market, they are not closely related to the culture of each company, and in order to highlight the specialty of uniform, 
some companies spare no expense and have their uniform custom made with unique fabric. For example, the British designer Bruce Oldfield designed brand-new staff uniform for 1200 McDonald's restaurants in Britain: the waitress will wear blouse with patterns, matched with fashionable black overskirt, and they also wear matching brown tie. The most special design is the decoration pattern, which uses the "M" logo of McDonald's, which is similar to the application of the logo of Louis Vuitton.

\section{Development trend of fabric in uniform design}

Due to requirement by the work environment, the special uniform fabrics have become a focus during the technical development of uniform, and they are obtained after multiple scientific researches and experiments, which can protect the personal safety under special work environment through various functions, such as flame retardant, withstanding voltage, acid proof, alkali proof, oil proof, water proof, ultraviolet proof and radiation proof performances.

\section{Radiation-proof fabric}

More and more electronic and electric devices tend to generate electromagnetic wave with different energies during the operation. For the good conductor of human body, the electromagnetic wave will unavoidably cause various degrees of damage. Under such environment, the staff will wear uniform made with radiation-proof fabric to reduce the damage to their health.

There are mainly two types of radiation-proof fabric: one type wraps cotton yarn outside of the stainless steel filament, which has great breathability and laundering durability, and because the metal wire has a small resistance, which can shield and absorb the electromagnetic wave; for another type, the fabric surface is coated with conductive and absorbing metal ions, and he radiation-proof performance of this kind of material can reach $60 \mathrm{~dB}$, but it has poorer breathability and laundering durability. The radiation-proof fabrics generally adopt the spray coating, which sprays the metallic paint onto the fabric to form flake shield.

\section{Flame-retardant and heat-insulation fabric}

The firefighter's protective clothing refers to the protective clothing worn by the firefighter when entering the fire scene to distinguish the fire, and the firefighter's protective clothing generally uses flame-retardant fabric. At present, in most foreign countries such as the United States, France, Japan and Britain, the aromatic polyamide fiber fabric is generally adopted, which has great flame-retardant performance, high strength and high thermal stability, the combustion products has 
extremely low toxicity, and this fabric also has certain acid and alkali proof ability; Nomex is the product of DUPONT USA, the Nomex fiber has great thermal stability, and it won't melt under $377^{\circ} \mathrm{C}$, but it will decompose; Kanox is a kind of preoxidized fiber, which is obtained through incomplete carbonization (the fiber can become fire resistant this way) of polyacrylonitrile fiber. It can resist the chemical substance, thermal radiation and melted metal, which has great thermal stability.

\section{Oil- and dirt-proof material}

This kind of fabric is mainly made of fluorocarbon polymer, which can form seamless and invisible protective film. This film can wrap the fabric fiber, which can be solidly bound to the fiber under the function amplification agent, the fabric will obtain unprecedented functional stability, while the breathability and moisture penetrability of fabric are not affected, and the various original performances of fabric can be optimized. After treatment, the fabric is nontoxic and nonirritant, which does not have any toxic and side effects caused by formaldehyde and heavy metal, and it is safe and environment friendly with a soft touch, which is also comfortable to ware.

\section{Moisture-absorption and penetrability fabric}

Some personnel are working outdoors most of the time, and under this circumstance, the uniform fabric should not only be very comfortable, but under a large amount of activities with profuse sweat, the fabric should not stick to the skin to generate the cold and humid feel. For natural fiber, take cotton for example, when people sweat profusely, the cotton fiber will absorb the moisture and expand, and the diffusing speed of moisture is also slow, which will generate a cold and humid feel in human body; for synthetic fiber, take polyester for example, it has small moisture absorption ability and poor moisture penetrability, due to accumulation of static electricity, it tends to cause problem when wearing the fabric, and especially during the activity, it could cause a feeling of stuffiness. Therefore, the polyester production and scientific research departments of various countries focus on improving the moisture absorption and penetrability performances of polyester.

At present, R \& D of this kind of fabric is mainly conducted through physical or chemical modification, or combing both methods. For example, for special profiled fiber, changing the form of spinneret orifice is a simple, direct and effective method to improve the moisture permeability of fiber, which will mainly generate many longitudinal grooves in the profiled fiber. Through the wicking effects of these grooves, the fiber can absorb the moisture and diffuse the sweat. The Coolmax polyester produced by DUPONT USA has a unique flat cross section, there are four 
longitudinal grooves on the fiber surface, and its size is $20 \%$ bigger than normal section, so its sweat diffusing performance is higher than the regular polyester; the styrene polymer blending or composite yarn blends the hydrophilic polymer and PET chip, in the meantime, the profile spinneret with special design is used to produce the moisture-absorbing and sweat-diffusing fiber, and the polyester chip and hydrophilic polymer are blended into profiled moisture-absorbing and sweat-diffusing fiber with skin core.

\section{Conclusion}

With the continuous development of technology, the future uniform design industry also advances in exploration. In the era with rapid development of science and technology, in order to become an outstanding uniform designer, we should recognize the development trend of this industry. During the design, efforts should be made to explore the development advantages of corporate customer, the uniform design should be integrated into the company culture from a stronger perspective, comprehensive individual packaging should be conducted to the company, so that the company can stand out among its peers, and the company culture of customer can be perfectly presented through the high-taste uniform. During each process of uniform design, we should keep pace with the times and promote the industrial and corporate development as the final goal of design.

\section{References:}

[1] Wang Gehui, Performance and Selection of Costume Fabric, Shanghai: Donghua University Press, May, (2013)

[2] Guo Fengzhi, Xing Shengyuan and Guo Ruiliang, Development of New Costume Fabric, Beijing: China Textile Press, September(2014)

[3] Chang Shuxiong and Wang Xiaoying, Professional Costume Design Course, Liaoning: Liaoning Fine Arts Publishing House, June(2014)

[4] Wang Fumei, Performance of Costume Fabric, Shanghai: Donghua University Press, December (2000) 